Z Gerontol Geriat 2011 [Suppl 2] · 44:101-112 DOI 10.1007/s00391-011-0246-6

Online publiziert: 2. Dezember 2011

(c) Springer-Verlag 2011

U. Thiem ${ }^{* 1,2} \cdot$ T. Hinrichs ${ }^{* 3} \cdot$ C.A. Müller* ${ }^{* 4} \cdot$ S. Holt-Noreiks ${ }^{* 5} \cdot$ A. Nag ${ }^{* 6}$.

C. Bucchi ${ }^{3}$. U. Trampisch ${ }^{3}$ - A. Moschny ${ }^{3} \cdot$ P. Platen ${ }^{3}$ - E. Penner ${ }^{4}$. U. Junius-Walker ${ }^{4}$. E. Hummers-Pradier ${ }^{4} \cdot$ G. Theile ${ }^{4}$. S. Schmiedl 5 . P.A. Thürmann 5 . S. Scholz ${ }^{6}$. W. Greiner ${ }^{6} \cdot$ R. Klaaßen-Mielke ${ }^{2} \cdot$ L. Pientka ${ }^{1} \cdot$ H.J. Trampisch ${ }^{2}$

${ }^{1}$ Klinik für Altersmedizin und Frührehabilitation, Marienhospital Herne, Klinik Börnig, Ruhr-Universität Bochum, Herne

${ }^{2}$ Abteilung für Medizinische Informatik, Biometrie und Epidemiologie, Ruhr-Universität Bochum

${ }^{3}$ Lehrstuhl für Sportmedizin und Sporternährung, Ruhr-Universität Bochum

${ }^{4}$ Institut für Allgemeinmedizin, Medizinische Hochschule Hannover

${ }^{5}$ Lehrstuhl für Klinische Pharmakologie, Fakultät für Gesundheit, Universität Witten/Herdecke

${ }^{6}$ Fakultät für Gesundheitswissenschaften, Arbeitsgruppe 5: Gesundheitsökonomie und Gesundheitsmanagement, Universität Bielefeld

\title{
Voraussetzungen für ein neues Versorgungsmodell für ältere Menschen mit Multimorbidität
}

\section{Ergebnisse und Schlussfolgerungen aus 3-jähriger Forschung im PRISCUS-Verbund}

Es ist allgemein anerkannt, dass die Bedürfnisse chronisch kranker Menschen in der traditionellen Gesundheitsversorgung eher unzureichend bedient werden $[3,6$, 13]. Eine Ursache dafür ist die eher krankheitszentrierte Vorgehensweise primärärztlicher Einrichtungen, in Deutschland der Hausarztpraxis $[6,56]$. Vorstellungen und Präferenzen des Patienten und der Angehörigen werden oft nicht beachtet. Patientenzentrierte Ziele, wie Symptomkontrolle und Lebensqualität, stehen zu selten im Vordergrund. Medikamentöse Therapie führt häufig zu Polypharmazie, bei der die Wahrscheinlichkeit von unerwünschten Wirkungen zunimmt, die Entfaltung erwünschter Wirkungen aber vielfach unsicher bleibt. Weitere Probleme, wie unzureichende oder widersprüchliche Leitlinienempfehlungen zu einzelnen Erkrankungen oder die
Compliance mit komplexen Therapieplänen, werden zu wenig hinterfragt $[6,56]$.

Einen möglichen Rahmen für eine Verbesserung der Versorgung bietet das sog. Chronic Care Model [3, 13], das ein interaktives System aus verschiedenen Versorgungskomponenten beschreibt. Die Komponente „Hilfe zur Entscheidungsfindung “ setzt die Kenntnis umsetzbarer Managementstrategien auf der Basis fachlicher, idealerweise evidenzbasierter Empfehlungen voraus. Informationen zu diesen Strategien sollen in den Versor-

Die mit * gekennzeichneten Autoren sind zu gleichen Teilen am Zustandekommen dieses Manuskripts beteiligt und sollten daher als gleichwertige Erstautoren angesehen werden. Finanzierung: Der Forschungsverbund PRISCUS wird gefördert durch das Bundesministerium für Bildung und Forschung (BMBF, Förderkennzeichen 01 ET0720 bis 01ET0724). die unter mindestens zwei chronischen Krankheiten leiden. 
gungsprozess eingebettet und in die Entscheidungsfindung von Therapeuten und Betroffenen eingebunden werden. Die Komponente „Unterstützung der Selbsthilfe" erfordert die Aufbereitung von Informationen für Patienten und Angehörige, um Eigenaktivitäten und ein Selbstmanagement zu fördern [3, 13]. Die Weltgesundheitsorganisation WHO hat eine internationale Adaptation des Chronic Care Model initiiert [13]. Auch eine deutsche Fassung liegt vor [21]. Eine inhaltliche Ausgestaltung für das Problem Multimorbidität steht allerdings national und international noch aus.

Der Forschungsverbund PRISCUS geht davon aus, dass die wesentliche Evidenz für neue Versorgungsmodelle multimorbider Patienten noch geschaffen werden muss. Die einzelnen Teilprojekte versuchen, eine wissenschaftliche Grundlage für die unterschiedlichen, für den Betroffenen bedeutsamen Bereiche der Epidemiologie, Diagnostik und Therapie zu legen. Einzelheiten zum Verbund und seinen sieben Teilprojekten wurden bereits an anderer Stelle publiziert [55]. Schwerpunkte der Teilprojekte, die im Folgenden verschiedene Ergebnisse präsentieren, sind: körperliche Aktivität als Teil des Selbstmanagements und zur Prävention eines funktionellen Abbaus, das geriatrische Assessment in der Hausarztpraxis sowie die individuelle Bewertung der Gesundheitsprobleme aus Perspektive des Patienten und seines Hausarztes, potenziell inadäquate Medikation und pharmakologische Interaktion als zentrale Probleme der medikamentösen Therapie sowie Auswirkungen von Multimorbidität auf Lebensqualität und Kosten.

\section{A. Körperliche Aktivität und Multimorbidität}

Die positiven Einflüsse regelmäßiger körperlicher Aktivität sowohl auf den Verlauf chronischer Erkrankungen, wie beispielsweise Diabetes mellitus und koronare Herzkrankheit, als auch auf den Erhalt von körperlicher Funktionsfähigkeit, Mobilität und Selbstständigkeit im Alter sind ausreichend dokumentiert [9]. Als Konsequenz aus den vorliegenden Erkenntnissen wird in aktuellen Empfehlungen zur körperlichen Aktivität im hö- heren Lebensalter ein multidimensionales Aktivitätsprogramm, das Kraft-, Ausdauer-, Balance- und Beweglichkeitstraining beinhaltet, propagiert [47]. Für die Gestaltung adressatengerechter Interventionsmaßnahmen ist ein tiefgehendes Verständnis des Aktivitätsverhaltens notwendig. Leider existieren für die deutsche Altenbevölkerung bislang nur wenige Daten zur genauen Art und zum Umfang der alltäglichen körperlichen Aktivität. Gemäß internationaler Daten scheinen insbesondere chronisch kranke und funktionell eingeschränkte Personen von mangelnder Aktivität und folglich weiterem Leistungsabbau betroffen zu sein [39]. Leben diese Menschen in eigener Wohnung, so sind sie schwer durch Interventionen zu erreichen. Das hausärztliche Umfeld könnte eine Möglichkeit darstellen, Aktivitätsprogramme an diese Zielgruppe heranzutragen [29].

\section{Ziele der Untersuchung}

Ziele des sportmedizinischen Teilprojekts im Forschungsverbund PRISCUS waren daher:

1. Entwicklung eines Fragebogens zur Erfassung der körperlichen Aktivität von Personen im Alter von 70 Jahren und älter (PRISCUS-Physical Activity Questionnaire, PRISCUS-PAQ)

2. Erfassung und Analyse von Daten zu körperlicher Aktivität in einer Kohorte älterer Hausarztpatienten (German Epidemiological Trial on Ankle Brachial Index, getABI [22])

3. Entwicklung eines Heimübungsprogramms für chronisch kranke und mobilitätseingeschränkte Ältere mit strukturierter Unterstützung durch die Hausarztpraxis (HOMEfit).

\section{Methoden und Ergebnisse}

\section{Fragebogen zur Erfassung körperlicher Aktivität}

Zur Gewinnung von Hinweisen zur Entwicklung eines Fragebogens zur Erfassung körperlicher Aktivität im höheren Lebensalter wurde zunächst eine Literaturübersicht erstellt [59]. Auf dieser Basis wurde der PRISCUS-PAQ für die Durchführung im Telefoninterview zur Erfassung der körperlichen Aktivität der ver- gangenen Woche entwickelt. Der Fragebogen erfasst in zehn Fragen Aktivitäten aus den Bereichen "Alltagsaktivitäten“ (Hausarbeit, Gartenarbeit, Wege zu Fuß), „sportbezogene Freizeitaktivitäten“ sowie „Inaktivität“. Die Reliabilität des Fragebogens wurde an 91 Personen (Alter 70 bis 98 Jahre, 36\% Männer) mit einem TestRetest-Abstand von 4 Wochen überprüft. Die Intraklassenkorrelation des PRISCUS-PAQ-Gesamtscores betrug 0,59 (95\%-Konfidenzintervall: 0,43-0,71); für die einzelnen Aktivitäten lag diese zwischen 0,47 (Wege zu Fuß) und o,82 (Fahrradfahren) [57]. Zur Prüfung der Validität des PRISCUS-PAQ wurde zudem an 114 älteren Personen die Korrelation zwischen PRISCUS-PAQ-Gesamtscore und der mittels Akzelerometer gemessenen körperlichen Aktivität ermittelt [58].

\section{Analyse von Daten zu körperlicher Aktivität}

Nachdem in der 5-Jahres-Verlaufsuntersuchung der getABI-Kohorte noch eine modifizierte Version des „Freiburger Fragebogens“ [19] zur Ermittlung der Sportpartizipation zum Einsatz gekommen war [30], konnte in der 7-JahresVerlaufsuntersuchung erstmals der PRISCUS-PAQ eingesetzt werden. Neben der körperlichen Aktivität in den Kategorien des PRISCUS-PAQ [44] wurden empfundene Barrieren [45] sowie hausärztliche Empfehlungen zur Steigerung der körperlichen Aktivität [28] erfragt. Die Analysen ergaben geschlechtsbezogene Unterschiede im Aktivitätsprofil (sportliche Aktivität vs. Hausarbeit). So verbrachten beispielsweise Männer mehr wöchentliche Zeit mit sportlicher Aktivität $(1,45$ vs. 1,10 h), während Frauen mehr Hausarbeit verrichteten (4,00 vs. 3,0o h; [44]). Gesundheitliche Probleme wurden als wesentliche Barriere zur Steigerung der körperlichen Aktivität identifiziert [43]. Zudem zeigte sich, dass nur etwa ein Drittel aller Befragten in den vorangegangenen 12 Monaten eine hausärztliche Empfehlung zu körperlicher Aktivität erhalten hatte. Hausärzte schienen ihre Empfehlung auf Patienten mit chronischen Erkrankungen zu fokussieren [28]. 


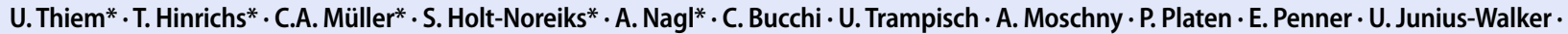
E. Hummers-Pradier · G. Theile · S. Schmied · P.A. Thürmann · S. Scholz · W. Greiner · R. Klaaßen-Mielke · L. Pientka · H.J. Trampisch Voraussetzungen für ein neues Versorgungsmodell für ältere Menschen mit Multimorbidität. Ergebnisse und Schlussfolgerungen aus 3-jähriger Forschung im PRISCUS-Verbund

\section{Zusammenfassung}

Hintergrund. Das gleichzeitige Auftreten oder Vorhandensein mehrerer chronischer Erkrankungen im Sinne einer Multimorbidität stellt den Betroffenen und seine Angehörigen, Ärzte und Therapeuten, aber auch das Gesundheitssystem vor große Herausforderungen. Für eine verbesserte medizinische Versorgung bietet sich das für chronisch Kranke entwickelte Chronic Care Modell an. Der Forschungsverbund PRISCUS versucht, die Voraussetzungen für ein daran orientiertes, neues Versorgungsmodell für multimorbide, ältere Patienten zu schaffen. Methoden und Ergebnisse. Übersichtsartig werden Ergebnisse aus vier der insgesamt sieben Teilprojekte des Forschungsverbunds dargestellt. Im sportmedizinischen Teilprojekt geht es um die Erfassung körperlicher Aktivi- tät über ein neues Fragebogeninstrument sowie die Entwicklung eines Heimübungsprogramms für chronisch kranke und in der Mobilität eingeschränkte Ältere. Das allgemeinmedizinische Teilprojekt befasst sich mit der Implementierung eines geriatrischen Assessments in der hausärztlichen Versorgung sowie dessen Auswirkungen. Im pharmakologischen Teilprojekt geht es um potenziell inadäquate Medikation für Ältere, Interaktionen und Nebenwirkungen. Das gesundheitsökonomische Teilprojekt eruiert Auswirkungen von Multimorbidität bei Älteren auf Lebensqualität und gesundheitsassoziierte Kosten.

Schlussfolgerung. Die Ergebnisse des Forschungsverbunds erlauben eine Abschätzung der Krankheitsfolgen von Multimorbi- dität und zeigen einige neue Ansätze zu Prävention, Diagnostik und Therapie bei Multimorbidität auf. Folgeprojekte werden sich mit der Wirksamkeit eines hausärztlich vermittelten, multidimensionalen Bewegungsprogramms sowie mit einem Interventionsprogramm zur Reduktion potenziell inadäquater Medikation bei Älteren befassen. Damit werden erste Voraussetzungen geschaffen, durch Anpassung der Versorgung nach Vorbild des Chronic Care Modell ein verbessertes Management von Patienten mit Multimorbidität zu erreichen.

Schlüsselwörter

Körperliche Aktivität · Geriatrisches Assessment · Medikation · Gesundheitsökonomie . Chronic Care Modell

\section{Prerequisites for a new health care model for elderly people with multiple morbidities.} Results and conclusions from 3 years of research in the PRISCUS consortium

\section{Abstract}

Background. The concurrent presence or manifestation of multiple chronic conditions, i.e. multimorbidity, poses a challenge to affected patients and their relatives, physicians, and practitioners, and to the health care system in general. Aiming to improve medical care for different chronic diseases, the Chronic Care Model also appears to be suited for multimorbidity. The established research consortium PRISCUS is trying to create some of the prerequisites for a new care model for multimorbid, elderly patients oriented along the lines of the Chronic Care Model.

Methods and results. Four out of seven subprojects of the research consortium provide an overview of some of their findings. Topics in a sports medicine subproject were the as- sessment of physical activity by means of a newly developed questionnaire and the development and feasibility testing of an exercise program for elderly people with chronic conditions and mobility impairment. Partners from family medicine implemented geriatric assessment in a primary care setting and evaluated its consequences. In a pharmacological subproject, potentially inappropriate medication as well as drug-drug interactions and dosing errors were addressed. The health economic subproject investigated quality of life impairment due to multiple chronic diseases and the effects of multimorbidity on costs.

Conclusions. The results of the PRISCUS research consortium allow a better description of consequences of multimorbidity and illustrate at least some new approaches towards prevention, diagnosis, and treatment of patients suffering from multimorbidity. Ongoing projects will test the efficacy of a physical activity program and a new complex intervention to reduce potentially inappropriate medication in the elderly. With this, the research consortium will create some prerequisites for a new health care model for patients with multimorbidity comparable to the Chronic Care Model.

Keywords

Physical activity - Geriatric assessment . Medication · Health economics · Chronic Care Model 


\section{Bewegungsprogramm \\ mit hausärztlicher Unterstützung (HOMEfit)}

Ergänzend zu den Analysen zur hausärztlichen Empfehlung in der getABI-Kohorte wurde eine Literaturanalyse zur Rolle des Hausarztes in der Vermittlung körperlicher Aktivität an ältere Patienten durchgeführt [25]. Diese ergab, dass der Hausarzt hierbei eine ganz zentrale Rolle einnehmen könnte. Allerdings besteht bislang keine gesicherte Evidenz hinsichtlich der Effektivität hausärztlicher Beratungsprogramme zu körperlicher Aktivität. Folglich empfehlen frühere Autoren $[2,36]$ zwar, den hausärztlichen Zugang zur Zielgruppe zu nutzen, verweisen allerdings auf die Notwendigkeit, den Hausarzt sowohl personell als auch mit zusätzlichen Kenntnissen (z. B. bezüglich der Anwendung von verhaltensändernden Strategien) zu unterstützen.

Auf Grundlage der gewonnenen Erkenntnisse sowie aktueller Leitlinien zur körperlichen Aktivität im hohen Lebensalter [47] wurde ein multidimensionales Bewegungsprogramm für chronisch kranke und mobilitätseingeschränkte Ältere entwickelt, das über eine neuartige Kooperation zwischen Hausärzten und Bewegungstherapeuten vermittelt wird (HOMEfit [27]). Der Hausarzt spielt hierbei eine wesentliche Rolle bei der Feststellung der gesundheitlichen Eignung und bei der Motivation der Patienten zu körperlicher Aktivität. Ein Bewegungstherapeut betreut die zugewiesenen Patienten während der 12-wöchigen Intervention in persönlichen Gesprächen in der Arztpraxis sowie telefonisch. In einer Pilotstudie mit 91 Patienten konnten Machbarkeit und Akzeptanz des Programms gezeigt werden [26].

\section{Schlussfolgerungen und Ausblick}

Mit dem PRISCUS-PAQ konnte ein Fragebogen entwickelt werden, der eine akzeptable Reliabilität im Vergleich mit international eingesetzten Aktivitätsfragebögen aufweist. Er eignet sich zur Erfassung der körperlichen Aktivität von älteren Personen in epidemiologischen Studien im deutschsprachigen Raum. In der 7-JahresVerlaufsuntersuchung der getABI-Kohorte kam der PRISCUS-PAQ erstmals in ei- ner epidemiologischen Studie zum Einsatz. So wurde zum einen eine Datenbasis zum Aktivitätsverhalten älterer Menschen in Deutschland geschaffen, zum anderen dienten die Erkenntnisse der Entwicklung einer aktivitätsfördernden Intervention. Diese macht sich das hausärztliche Setting zunutze, um die Zielgruppe der chronisch kranken und mobilitätseingeschränkten Älteren zu erreichen. Nachdem Machbarkeit und Akzeptanz der Intervention gezeigt werden konnten, soll nun eine randomisierte kontrollierte Studie die Wirksamkeit des Konzepts (u. a. auf funktionelle Fähigkeiten und Lebensqualität) untersuchen.

\section{B. Das hausärztliche Assessment für ältere Menschen: Gesundheitsprioritäten der Patienten, Versorgungsbedarf aus ärztlicher Sicht und resultierende Interventionen}

Die hausärztliche Medizin erfährt nicht nur in Deutschland durch den demographischen Wandel neue Herausforderungen. Bei einer zunehmend älter werdenden Bevölkerung werden chronische Erkrankungen und Multimorbidität häufiger festgestellt [6o]. Der Hausarzt spielt eine maßgebliche Rolle bei der medizinischen Versorgung älterer Menschen. Regelmäßigen Kontakt zum Hausarzt haben $96 \%$ der deutschen Senioren, ältere gesetzlich versicherte Patienten suchen ihren Arzt mehr als 10-mal pro Jahr auf, bei den über 9o-Jährigen verdoppelt sich diese Zahl auf über 22 Konsultationen [23]. Bei den im europäischen Vergleich relativ kurzen Konsultationen [12] kommen zumeist wenige Probleme oder sogar lediglich eine gerade aktuelle Frage zur Sprache. Vor diesem Hintergrund kann die Durchführung eines standardisierten umfassenden geriatrischen Assessments (GA) dem Arzt einen Überblick über die Gesundheitsprobleme seiner Patienten ermöglichen und zur Entwicklung eines abgestimmten Behandlungsplans wesentlich beitragen. Einige europäische Länder wenden ein solches GA in der ambulanten Versorgung an: England (Department of Health [11]) und Dänemark [61], in Deutschland wird es in der ambulanten Versorgung nur sehr selten und un- systematisch eingesetzt [54]. Mehrere Untersuchungen über den Effekt des GA in der hausärztlichen Praxis zeigten widersprüchliche Ergebnisse hinsichtlich Mortalität, zu Hause leben und Krankenhausaufnahmen $[17,53]$. Einige Studien konnten positive Einflüsse auf die gesundheitsbezogene Lebensqualität $[10,17]$ und soziale Interaktionen [17] nachweisen.

\section{Ziele der Untersuchung}

Das Hauptziel der Studie war, bei mobilen älteren Patienten in der hausärztlichen Praxis den Effekt eines standardisierten GA in Kombination mit der folgenden Konsultation hinsichtlich des Einflusses auf die subjektive Krankheitslast zu testen. Krankheitslast wurde hierbei definiert als Mittelwert der Bewertung der drei Dimensionen „Wichtigkeit“, „Belastung“, „Behinderung “ für das jeweilige aufgedeckte Gesundheitsproblem; die Mittelung aller problembezogener Mittelwerte eines Patienten ergaben einen Indexwert. Nebenziele waren die Beschreibung von Multimorbidität in der hausärztlichen Versorgung und der Vergleich der Wichtigkeitseinschätzungen der aufgedeckten Gesundheitsprobleme aus der Sicht von Patienten und Ärzten. Zudem wurde untersucht, welche Interventionen nach dem standardisierten GA (Standardized Assessment of Elderly People in Primary Care, STEP) und Konsultation geplant und letztlich durchgeführt wurden und welche Faktoren deren Umsetzung beeinflussen. In einer eingebetteten qualitativen Studie wurden der Ablauf und die Inhalte der STEP folgenden Konsultationen ausgewertet.

\section{Methoden}

Es wurde eine kontrollierte Interventionsstudie mit über 72-jährigen Hausarztpatienten und ihren Ärzten durchgeführt. Das standardisierte Assessment für ältere Menschen in der hausärztlichen Praxis (STEP), das in dieser Studie eingesetzt wurde, war in einer konzertierten europäischen Aktion Ende der 1990er Jahre entwickelt [48] und in Hausarztpraxen in Deutschland und England erfolgreich hinsichtlich der Machbarkeit getestet worden [37,38]. Das STEP-Assessment 
enthält 44 Items bezüglich relevanter Altersprobleme, die sich fünf Problemgruppen zuordnen lassen: dem somatischen, psychischen, funktionellen und sozialen Bereich sowie Risikofaktoren (- Tab. 1). In der ca. 45-minütigen Befragung durch Studienassistentinnen geben die Patienten selbst Auskunft über ihr Befinden, ergänzt durch wenige einfache Tests (zu Kognition und Sturzgefährdung) und Untersuchungen (Blutdruckmessung und Fußinspektion). Am Beginn der Studie wurde STEP in der Interventionsgruppe bei 436 Patienten in 46 Hausarztpraxen in Hannover und Umgebung sowie in der Kontrollgruppe bei 396 Patienten in 28 Praxen im Ruhrgebiet (getABI-Kohorte [22], Wittener Ärztenetz) angewendet. Sämtliche Patienten bewerteten im Anschluss an STEP die Krankheitslast eines jeden aufgedeckten Gesundheitsproblems. Lediglich die Interventionspatienten besprachen die Ergebnisse anschließend mit ihrem Hausarzt; die Kontrollgruppe erhielt nach dem Assessment und während der 3-monatigen Nachbeobachtung die „übliche Behandlung“, ohne dass die Hausärzte das Assessmentergebnis erfuhren. Nach dieser Zeitspanne bewerteten sämtliche Teilnehmer (abzüglich 96 Drop-outs), diesmal in einem Telefoninterview, die initialen Gesundheitsprobleme in Hinblick auf die Krankheitslast. Zusätzlich wurden die Hausärzte der Interventionsgruppe zu Beginn nach ihrer Bewertung der Wichtigkeit der Gesundheitsprobleme ihrer Patienten befragt. Sie gaben darüber hinaus an, für welche aufgedeckten Probleme sie Maßnahmen (Diagnostik, Therapie) planten. Nach dem Follow-up teilten sie mit, welche der geplanten Maßnahmen zwischenzeitlich tatsächlich umgesetzt worden waren. Die Auswertung der Daten erfolgte mittels SAS, Version 9.1.

Für die eingebettete qualitative Untersuchung der Konsultationen nach STEP wurden in der Interventionsgruppe 33 Videoaufnahmen dieser Arzt-PatientGespräche angefertigt. Durch „theoretical sampling" hinsichtlich Alter und Geschlecht von Patienten und ihren Hausärzten wurde eine Stichprobe von 24 Aufnahmen ausgewählt. Mit einem über offenes Kodieren von 6 Aufnahmen (Software atlas.ti) erstellten Kategoriensystem wur-

\begin{tabular}{|c|c|}
\hline Gruppe & Gesundheitsprobleme \\
\hline Körperliche Gesundheit & $\begin{array}{l}\text { Alterserkrankungen } \\
\text { Alterssyndrome } \\
\text { Schmerz } \\
\text { Sensorik } \\
\text { Orientierungstest }\end{array}$ \\
\hline Psychische Gesundheit & $\begin{array}{l}\text { Depression } \\
\text { Trauer } \\
\text { Einsamkeit } \\
\text { Angst }\end{array}$ \\
\hline Funktioneller Zustand & $\begin{array}{l}\text { Aktivitäten des täglichen Lebens (ADL/IADL) } \\
\text { Gangtest }\end{array}$ \\
\hline Soziales & $\begin{array}{l}\text { Wohnung: Reparaturbedarf, Hindernisse } \\
\text { Hilfe im Notfall/Krankheit } \\
\text { Zu pflegende Angehörige } \\
\text { Finanzielle Situation }\end{array}$ \\
\hline Risikofaktoren & $\begin{array}{l}\text { Bewegung } \\
\text { Ernährung } \\
\text { Rauchen } \\
\text { Alkohol } \\
\text { Impfschutz } \\
\text { Probleme Medikamenteneinnahme }\end{array}$ \\
\hline
\end{tabular}

de die Videostichprobe inhaltsanalytisch hinsichtlich des zeitlichen Ablaufs, der thematischen Einteilung und der Art der Entscheidungsfindung untersucht (Software Videograph, IPN, Rolf Rimmele). Im Weiteren erfolgte eine Bewertung zur Patientenzentriertheit.

\section{Ergebnisse und Diskussion}

\section{Veränderung der Krankheitslast}

Hinsichtlich des primären Outcomeparameters ließ sich in der Studie nachweisen, dass es zu einer leichten, aber signifikanten Senkung der mittleren subjektiven Krankheitslast in der Interventionsgruppe im Vergleich zur Kontrollgruppe kam. Somit konnte gezeigt werden, dass die Durchführung von STEP mit anschließender Konsultation des Arztes den Patienten zu einem besseren Umgang mit ihren Gesundheitsproblemen verhalf.

\section{Multimorbidität}

Das STEP-Assessment deckte durchschnittlich 11,4 (Spanne 2-29) Probleme je Patienten der Gesamtgruppe $(n=738)$ auf. Frauen hatten in dieser Gruppe durchschnittlich mehr Gesundheitsprobleme als Männer (12,6 zu 9,7). Über 8o-Jährige nannten mehr Probleme als die jüngeren Teilnehmer: Durchschnittlich wurden bei ihnen 11,9 Probleme ge- funden, bei den 72- bis 79-Jährigen waren es 11,1 Probleme.

Ausgewertet nach der Häufigkeit der einzelnen Gesundheitsprobleme gaben mehr als drei Viertel der Teilnehmer der Gesamtgruppe einen Bluthochdruck oder einen fraglichen oder unzureichenden Impfschutz hinsichtlich Tetanus, Diphtherie, Influenza oder Pneumokokken an oder berichteten über Schmerzen. Mehr als jeder zweite Patient hatte Schwierigkeiten bei der Einnahme seiner Medikamente, erhöhte Cholesterinwerte in der Vorgeschichte oder Probleme mit seinen Füßen (• Tab. 2). Zu den häufigsten Gesundheitsproblemen zählten somit in dieser Studie, wie auch in anderen Erhebungen [23], die klassischen chronischen Alterserkrankungen Bluthochdruck und Hypercholesterinämie, zudem wurden von den älteren Patienten sehr häufig Schmerzen angegeben. Die hohe Prävalenz eines fehlenden oder fraglichen Impfschutzes ergibt sich u. a. aus dem Umstand, dass schon eine einzelne fehlende bzw. fragliche Impfung bei vier impfpräventablen Erkrankungen zu einem Triggern des Items führte. Interessanter ist die hohe Prävalenz von Fußerkrankungen im Alter; eine regelmäßige Einbeziehung der Füße in die Untersuchung älterer Menschen erscheint sehr sinnvoll. Polypharmazie im Alter gerät in den letzten Jah- 
Tab. 2 Rangfolge der häufigsten Gesundheitsprobleme (Gesamtgruppe n=738)

\begin{tabular}{|llll} 
Rang & Gesundheitsproblem & Absolute Häufigkeit & $\begin{array}{l}\text { Relative Häufigkeit } \\
(\%)\end{array}$ \\
1 & Bluthochdruck & 597 & 80,9 \\
1 & Unzureichender/fraglicher Impfschutz & 597 & 80,9 \\
3 & Schmerzen & 584 & 79,1 \\
\hline 4 & Probleme bei Medikamenteneinnahme & 483 & 65,4 \\
\hline 5 & Hypercholesterinämie & 474 & 64,2 \\
\hline 6 & Fußprobleme & 423 & 57,3 \\
\hline 7 & Unzureichende körperliche Betätigung & 334 & 45,3 \\
\hline 8 & Schlafstörungen & 286 & 38,8 \\
\hline 9 & Trauer & 277 & 37,5 \\
\hline 10 & Auffälliger Orientierungstest & 229 & 31,0 \\
\hline
\end{tabular}

ren zunehmend in den Fokus der Versorgungsforschung, der Stellenwert dieses Themas wird durch die in dieser Studie häufig angegebenen Probleme bei der Medikamenteneinnahme (meist durch Einnahme von mehr als 5 Medikamenten bedingt) bestätigt.

\section{Wichtigkeitsbewertungen}

Die Beurteilung der Wichtigkeit der individuellen Gesundheitsprobleme durch die Patienten beider Studienarme ermöglichte es uns, eine „Rangfolge der Wichtigkeit"von Gesundheitsproblemen durchzuführen. In der Gesamtgruppe war den betroffenen Patienten am wichtigsten, wenn sie Angehörige zu pflegen hatten. Des Weiteren zählten drei psychische Probleme (Angst, Einsamkeit, gedrückte Stimmung) zu den sechs wichtigsten Gesundheitsproblemen. Eine schwierige finanzielle Situation wurde am viertwichtigsten eingestuft. Somatische Probleme folgten erst danach. In der Interventionsgruppe veränderte sich die Wichtigkeitsbewertung über alle Krankheitsprobleme im Laufe der 3 Monate signifikant im Vergleich zur Kontrollgruppe. Die Patienten in der Interventionsgruppe schätzten ihre Gesundheitsprobleme nun im Durchschnitt als geringfügig weniger wichtig ein, sie konnten besser mit ihnen umgehen. Der Kontrollgruppe wurden ihre Probleme wichtiger. Beim Vergleich der Wichtigkeitsbewertungen durch Arzt und Patient in der Interventionsgruppe zu Studienbeginn fiel auf, dass es hier zu einer unterschiedlichen Hierarchisierung kam. Zudem vergaben die Patienten für ihre zehn wichtigsten Items jeweils höhere Werte als die Ärzte für die ihren. Ver- mutlich ist die höhere Wichtigkeit durch die direkte Betroffenheit der Patienten zu erklären.

Die Auswertung der Neuaufdeckungen sowie der geplanten und durchgeführten Interventionen steht noch aus.

Die Besprechung der STEP-Ergebnisliste in der Konsultation nach STEP dauerte im Mittel 9,45 min, von der Gesamtzahl der durch STEP aufgedeckten Probleme wurden hierbei über $95 \%$ besprochen. Eine gesonderte Interventionsplanung nahm im Mittel 1,51 min in Anspruch.

Knapp 70\% der teilnehmenden Ärzte der Interventionsgruppe fanden, dass sich STEP gut zur problemübergreifenden Behandlungsplanung eigne, sogar über drei Viertel schätzten es als gut geeignet zur Neuaufdeckung altersrelevanter Problem ein. Allerdings waren auch knapp drei Viertel der Meinung, dass es hinsichtlich Zeit und Aufwand in der Praxis nicht gut durchführbar sei.

\section{Schlussfolgerungen}

Es konnte nachgewiesen werden, dass STEP zusammen mit einer anschließenden gemeinsamen Besprechung der Ergebnisse von Patient und Arzt zu einer Senkung der subjektiven Krankheitslast führt. Der Austausch kann hierbei zu einem umfassenden Blick auf die Gesundheitsbedürfnisse des Patienten führen und zu einer vermehrten Einbeziehung des Patienten auch im Sinne einer partizipativen Entscheidungsfindung. Die Wichtigkeitsbewertung der Probleme gibt ebenfalls wesentliche Einblicke in die Patientenperspektive und zeigt, dass sich die- se von der ärztlichen Perspektive oft deutlich unterscheidet. Das STEP-Assessment wurde im Rahmen der Studie (von Studienassistentinnen durchgeführt) von den Ärzten gut angenommen; dennoch wurde es in der hier verwendeten Form als zu aufwendig für eine routinemäßige Durchführung in der Regelversorgung bewertet. Ein Ziel des Folgeprojekts ist deshalb die Erstellung eines kurzen, modularen, auch in der Hausarztpraxis machbaren Assessments für ältere Patienten.

\section{Multimorbidität und Polypharmazie: \\ Analyse von inadäquater \\ Medikation, Interaktionen und Nebenwirkungen}

\section{Ziele und Methoden}

Im Rahmen des pharmakologischen Teilprojekts (Multimorbidität und Polypharmazie: Analyse von Interaktionen, inadäquater Medikation und Nebenwirkungen) wurde mithilfe eines Expertenkonsens die PRISCUS-Liste potenziell inadäquater Medikamente (PIM) für ältere Menschen entwickelt [34]. Hierbei handelt es sich um Arzneimittel, deren Anwendung allgemein bei älteren Patienten nicht geeignet ist, die bei bestimmten Erkrankungen nicht zu verwenden sind oder deren Dosierungen angepasst werden müssen, um unerwünschte Wirkungen zu vermeiden [15]. Die Erstellung der PRISCUS-Liste erfolgte in Anlehnung an internationale Arbeiten [1, 15, 40, 43], angepasst an das deutsche Verordnungsverhalten und den hiesigen Arzneimittelmarkt. Es erfolgte eine Expertenbefragung (Delphi-Methode) zur Erstellung der finalen PIM-Liste für Ältere (PRISCUS-Liste). Insgesamt bewerteten 27 Experten in zwei Befragungsrunden 83 Medikamente als möglicherweise ungeeignet für ältere Patienten. Für einige der Arzneimittel gelten Dosierungs-, Freisetzungsform- und Indikationsbeschränkungen. Neben Therapiealternativen und zu vermeidenden Komorbiditäten beinhaltet die PRISCUS-Liste Monitoringparameter und Dosierungshinweise für den Fall, dass die Nutzung eines PIM nach der PRISCUS-Liste nicht vermeidbar ist ([34]; s. auch http://www. priscus.net). 
Im Rahmen der drei im Verbund PRISCUS durchgeführten Kohortenstudien (getABI-, STEP-Studie, Schlaganfallregister) wurden in Patienteninterviews neben Erkrankungen, Arztkontakten und Krankenhausaufenthalten verschiedene Aspekte der Arzneimitteltherapie erfasst. Es wurden sämtliche von den Patienten genutzte Medikamente, deren Dosierung und Einnahmehäufigkeit ermittelt. Außerdem beantworteten die Patienten Fragen zur Compliance und zu verschiedenen Beschwerden bzw. Nebenwirkungen. Die Auswertung der Medikationsdaten erfolgte im Hinblick auf PIM, Arzneimittelinteraktionen (in Kooperation mit der Abteilung Klinische Pharmakologie und Pharmakoepidemiologie der Medizinischen Universitätsklinik Heidelberg) und mögliche unerwünschte Arzneimittelwirkungen. Der Zusammenhang mit PIM nach der PRISCUS-Liste und unerwünschten Ereignissen wie Krankenhausaufnahmen, häufigen Arztbesuchen und subjektiv berichteten Nebenwirkungen wurde evaluiert.

\section{Ergebnisse}

Arzneimitteldaten von 1936 Patienten der getABI-Kohorte, 737 Patienten der STEPStudie und 756 Patienten des Schlaganfallregisters konnten analysiert werden [35]. Die befragten Patienten aller drei Untersuchungen nahmen durchschnittlich $6 \pm 3$ Arzneimittel pro Tag ein, ca. zwei Drittel der Patienten der Kohorten nutzen sogar 5 oder mehr Medikamente (- Tab.3). Die häufigsten Medikamente der Patienten der getABI-Kohorte und der STEP-Studie waren Arzneimittel mit Wirkung auf das Renin-Angiotensin-System $(64,7$ bzw. 61,7\%). Bei den Schlaganfallpatienten waren diese Mittel die zweithäufigste Arzneimittelklasse, 62,8\% der Schlaganfallpatienten nutzen sie. Am häufigsten gaben die Schlaganfallpatienten entsprechend ihrer Vorerkrankung den Gebrauch antithrombotischer Arzneimittel an (88,5\%).

Der Anteil an Patienten mit PIM nach der PRISCUS-Liste ist in allen drei untersuchten Kohorten mit etwa 18\% vergleichbar (- Tab. 3). Die häufigsten Arzneistoffklassen (3. Ebene der ATC-Klassifizierung) mit möglicherweise ungeeigne-

\begin{tabular}{|c|c|c|c|}
\hline & getABI-Kohorte & STEP-Studie & Schlaganfallkohorte \\
\hline $\begin{array}{l}\text { Anzahl Patienten } \\
\text { (für Medikationsauswertung) }\end{array}$ & 1936 & 737 & 756 \\
\hline Anteil Frauen & $53 \%$ & $60 \%$ & $40 \%$ \\
\hline Alter & $78 \pm 4$ Jahre & $78 \pm 4$ Jahre & $70 \pm 11$ Jahre \\
\hline $\begin{array}{l}\text { Patienten mit regelmäßigem } \\
\text { Medikamentengebrauch }\end{array}$ & $98 \%$ & $99 \%$ & $96 \%$ \\
\hline $\begin{array}{l}\text { Durchschnittliche Anzahl an } \\
\text { Medikamenten }\end{array}$ & $6 \pm 3$ & $6 \pm 3$ & $6 \pm 3$ \\
\hline Patienten mit $\geq 5$ Medikamenten & $66 \%$ & $61 \%$ & $63 \%$ \\
\hline $\begin{array}{l}\text { Patienten mit potenziell inad- } \\
\text { äquaten Medikamenten nach } \\
\text { PRISCUS-Liste }\end{array}$ & $17 \%$ & $18 \%$ & $18 \%$ \\
\hline
\end{tabular}

Tab. 4 Häufigste und klinisch relevante Interaktionen in der getABI-Kohorte

\begin{tabular}{|c|c|c|}
\hline Arzneimittel 1 & Arzneimittel 2 & Konsequenz \\
\hline NSAID & $\begin{array}{l}\text { ASS bzw. Clopidogrel } \\
\text { Phenprocoumon }\end{array}$ & Gefahr gastrointestinaler Blutungen \\
\hline Phenprocoumon & $\begin{array}{l}\text { Amiodaron } \\
\text { Tamoxifen } \\
\text { Cotrimoxazol und andere } \\
\text { Antibiotika }\end{array}$ & Blutungsgefahr \\
\hline Amiodaron & $\begin{array}{l}\text { Herzglykoside } \\
\text { Verapamil }\end{array}$ & Exzessive Bradykardie, Digitoxinintoxikation \\
\hline Simvastatin & $\begin{array}{l}\text { Amiodaron } \\
\text { Clarithromycin } \\
\text { Calciumantagonisten }\end{array}$ & $\begin{array}{l}\text { Teilweise kontraindiziert, } \\
\text { z. T. max. } 10 / 20 \text { mg Simvastatin } \rightarrow \text { Myopa- } \\
\text { thiegefahr }\end{array}$ \\
\hline NSAID & $\begin{array}{l}\text { ACE-Hemmer } \\
\text { Schleifendiuretika }\end{array}$ & Nierenfunktionsstörung \\
\hline ACE-Hemmer & Allopurinol & Blutbildungsstörungen \\
\hline Clopidogrel & $\begin{array}{l}\text { Protonenpumpeninhibitor, } \\
\text { insbesondere Omeprazol } \\
\text { Esomeprazol }\end{array}$ & Wirkverlust von Clopidogrel \\
\hline Methotrexat & $\begin{array}{l}\text { NSAID } \\
\text { Acetylsalicylsäure }\end{array}$ & Methotrexattoxizität \\
\hline
\end{tabular}

ten Medikamenten sind in der getABIund in der Schlaganfallkohorte Antidepressiva (Psychoanaleptika, No6), Herztherapeutika (Co1) und Anxiolytika/Sedativa/Neuroleptika (Psycholeptika, No5). Bei der STEP-Studie sind lediglich die Plätze 2 und 3 vertauscht. (Reihenfolge No6, No5 und Co1). Auf Wirkstoffebene dominieren bei den Herz-/Kreislauf-Medikamenten Acetyldigoxin, Doxazosin und Sotalol, bei den psychotropen Substanzen Amitriptylin, Bromazepam und Doxepin. In der Schlaganfallkohorte ist der hohe Gebrauch von Vasodilatatoren wie Piracetam und Naftidrofuryl auffällig.

In Übereinstimmung mit einigen Studien $[16,20]$ und gleichzeitig im Unterschied zu anderen internationalen Untersuchungen $[4,7,41]$ wurde kein statis- tisch signifikanter Zusammenhang zwischen der PIM-Nutzung und höherem Alter oder Geschlecht in der multivariaten Analyse festgestellt.

Patienten mit potenziell inadäquaten Medikamenten nach der PRISCUSListe sind häufiger von Polypharmazie ( $\geq_{5}$ Arzneimittel) betroffen als Patienten ohne PIM $(\mathrm{p}<\mathrm{o}, \mathrm{oo1})$. Die univariaten und multivariate Analysen der Daten der STEP- und getABI-Kohorte konnten einen Zusammenhang zwischen dem Gebrauch von PIM und häufigen Arztbesuchen $(\geq 5$ Arztbesuche in den letzten 3 Monaten) zeigen. Ein Zusammenhang mit Krankenhausaufenthalten wurde nicht festgestellt. In der univariaten Analyse der getABI-Kohorte bestand noch ein Zusammenhang zwischen PRISCUS- 
PIM und mehr als 2 Stürzen in den letzten 12 Monaten.

Die Auswertung der Arzneimittelinteraktionen erfolgte in Kooperation mit der Medizinischen Universitätsklinik Heidelberg (Abteilung Klinische Pharmakologie und Pharmakoepidemiologie). In der getABI-Kohorte ergab sich eine Vielzahl von häufig auftretenden und relevanten Interaktionen (• Tab.4). Bei 1937 Patienten wurden insgesamt 16 kontraindizierte, 577 schwerwiegende, 2307 mittelschwere und 1014 leichte Interaktionen ermittelt. Für 22\% aller Befragten konnte mindestens eine schwerwiegende oder kontraindizierte Interaktion in der Dauermedikation entdeckt werden. Für die Schlaganfallkohorte ergaben sich sehr ähnliche Ergebnisse, hier wurden bei $25 \%$ der Befragten Interaktionen dieser Relevanz eruiert.

\section{Diskussion}

Obgleich sich zahlreiche Argumente gegen „Negativlisten“ aufführen lassen, ist es in diesem Teilprojekt gelungen, einen bundesweiten Expertenkonsens zum Thema „potenziell inadäquate Medikation für ältere Menschen“ herbeizuführen. Die Grenzen und Limitationen der PRISCUSListe wurden ausführlich diskutiert [34]. Die PRISCUS-Liste als Werkzeug zur Vermeidung dieser Arzneimittel wurde mittlerweile von verschiedenen Anbietern für Verordnungssoftware implementiert und wird als Grundlage für ärztliche Fortbildungen und Therapiezirkel genutzt.

Die Analyse der Medikation der Kohorten ergibt eine PIM-Prävalenz von etwa $18 \%$. Dies stimmt mit internationalen Daten überein [14]. Es konnte nur ein schwacher Zusammenhang zwischen PIM-Gebrauch und Stürzen festgestellt werden, was möglicherweise an der relativ geringen Gebrechlichkeit der Teilnehmer liegen kann; der Anteil von Patienten mit einer Pflegestufe lag in den getABI- und STEP-Kohorten unter 3\%. Andererseits ist die Anwendung von PIM assoziiert mit mehrfachen Arztbesuchen und schwach assoziiert mit einer etwas schlechteren Lebensqualität als bei Nichtanwendung von PIM. In der Zusammenschau mit der Literatur lässt sich die Hypothese ableiten, dass die Vermeidung von PRISCUS-PIM bei einem entsprechend vorselektierten
Patientenkollektiv mit einer Reduktion von unerwünschten Ereignissen einhergeht.

Im Hinblick auf Multimorbidität, Polypharmazie und Interaktionen fand sich ein erstaunlich hoher Anteil von Arzneimittelwechselwirkungen in den analysierten Kohorten. Auch hier könnte eine entsprechende Modifikation der Multimedikation zur Erhöhung der Arzneimitteltherapiesicherheit bei Senioren beitragen. Diese Befunde zur Pharmakotherapie in den untersuchten Kohorten des PRISCUS-Verbunds bilden die Grundlage für die Intervention der im Folgeprojekt geplanten Studie zur Reduktion potenziell inadäquater Medikation bei Aelteren (RIME-Studie).

\section{Lebensqualität und gesund- heitsökonomischen Aspekte von Multimorbidität im Alter}

Veränderte Morbiditätsmuster in westlichen Industrienationen führen zu einem grundlegenden Wandel der Anforderungen an die Patientenversorgung. Aufgrund der Zunahme von langjährigen chronischen (Mehrfach-)Erkrankungen steht dabei nicht mehr ausschließlich die vollständige Heilung, sondern zunehmend eine Linderung von Beschwerden und eine Sicherstellung der Lebensqualität im Fokus der Bemühungen. Kenntnisse über Auswirkungen verschiedener Morbiditätsmuster auf die gesundheitsbezogene Lebensqualität älterer Personen sind von besonderer Relevanz, um den Leistungserbringern ein umfassendes Bild vom Zustand bzw. der Wahrnehmung ihrer älteren, oftmals multimorbiden Patienten zu verschaffen und so, über zielgerichtete Interventionen, eine bedarfsgerechte Versorgung zu erreichen.

Die Gruppe alter und hochaltriger Menschen leidet allerdings nicht nur am meisten unter dem simultanen Auftreten (chronischer) Erkrankungen, sondern stellt gleichzeitig eine relativ kleine Vielnutzergruppe innerhalb des Gesundheitssystems dar, die einen Großteil der Kosten verursacht [8]. Dies wird für den deutschen Versorgungskontext insbesondere durch Zahlen des statistischen Bundesamts deutlich, wonach die Bevölkerung über 65 Jahre lediglich 19\% der Gesamtbe- völkerung ausmacht, allerdings für 47,1\% der Gesundheitsausgaben verantwortlich ist [49]. Es ist zu vermuten, dass sich der finanzielle Druck im Gesundheitswesen durch den prognostizierten Anstieg von 16 Mio. (2006) auf ca. 23 Mio. Menschen über 64 Jahre im Jahr 2060 weiter verschärfen wird [50]. Um die Versorgung für ältere und multimorbide Personen optimieren und Verteilungsentscheidungen für knappe Ressourcen im Gesundheitswesen treffen zu können, ist es notwendig, detaillierte Informationen über die Auswirkungen verschiedener Multimorbiditätsmuster auf die gesundheitsassoziierten Kosten zu generieren.

\section{Auswirkungen von Multimorbidität auf die gesundheitsbezogene Lebensqualität}

Im Rahmen einer systematischen Übersichtsarbeit wurden 32 Studien identifiziert, die, mithilfe unterschiedlicher Messinstrumente, Lebensqualität in Verbindung mit der Morbiditätssituation messen. Als zentrales Ergebnis zeigt sich, dass die Lebensqualität nicht grundsätzlich im Alter, sondern vornehmlich bei Vorliegen von Mehrfacherkrankungen absinkt. Mit zunehmendem Alter vermehren sich die multimorbiden Zustände, sodass auf diese Weise indirekt Auswirkungen auf die Lebensqualität entstehen. In 24 Studien wurde ein Absinken der Lebensqualität bei einer steigenden Zahl von Krankheiten beobachtet - vor allem im Bereich der physischen Dimension der gesundheitsbezogenen Lebensqualität. Bei der Betrachtung einzelner Krankheitsbilder zeigen vor allem Muskel-Skelett-Erkrankungen, wie beispielsweise Arthritis oder Rückenbeschwerden, aber auch Herz-KreislaufErkrankungen, einen besonders starken negativen Einfluss auf die gesundheitsbezogene Lebensqualität. In der mentalen Dimension ist vor allem die Depression als besonders einschränkender Faktor der Lebensqualität hervorzuheben [31].

In einer ersten Analyse der getABI-Kohorte zu den Auswirkungen von Mehrfacherkrankungen und gesundheitsassoziierten Ereignissen auf die gesundheitsbezogene Lebensqualität wurden die Ergebnisse der beiden generischen Lebensqualitätsmessinstrumente EQ- 
$5 \mathrm{D}$ und SF-8 von 2120 älteren Personen $(76,29 \pm 4,48$ Jahre) verwendet. Der EQ${ }_{5} \mathrm{D}$ der EuroQoL-Gruppe ist ein Indexinstrument und beinhaltet die fünf Dimensionen „Beweglichkeit/Mobilität“, „für sich selbst sorgen“, „alltägliche Aktivitäten“, „Schmerzen/körperliche Beschwerden“ sowie „Angst/Niedergeschlagenheit“, deren Einzelergebnisse durch ein validiertes Verfahren in einen Indexwert überführt werden. Der SF-8 hingegen ist ein sog. Profilinstrument, dessen Ergebnisse die Erstellung von Gesundheitsprofilen mit den acht Items ,allgemeine Gesundheitswahrnehmung“, „körperliche Funktionsfähigkeit“, „körperliche Rollenfunktion“, „Schmerz“, „Vitalität“, „soziale Funktionsfähigkeit“, „emotionale Rollenfunktion" und "psychisches Wohlbefinden“ ermöglichen. Diese Ergebnisse lassen sich weiter zu einer mentalen und einer physischen Summenskala zusammenfassen [32].

Die deskriptiven Auswertungen zeigen ein klar inverses Verhältnis zwischen der Anzahl an Grunderkrankungen bzw. gesundheitsbeeinflussenden Ereignissen und der Lebensqualität bei älteren Personen. Die negative Beeinflussung des EQ5D-Gesamtwerts und der körperlichen Subskala des SF-8 fällt dabei stärker aus als die der mentalen Dimension. Die Analyse des Einflusses von einzelnen Erkrankungen auf die Lebensqualität zeigt, dass Personen mit chronischer peripherer arterieller Verschlusskrankheit (PAVK) oder Beeinträchtigungen des Bewegungsapparats sowie Personen, die in den letzten 2 Jahren ein zerebro- oder kardiovaskuläres Ereignis erlitten, besonders niedrige Lebensqualitätswerte (gemäß EQ-5D und/ oder SF-8 körperlich) aufweisen. Außerdem ist bemerkenswert, dass einige Personengruppen mit objektiv schwerwiegenden Gesundheitsproblemen (z. B. Krebserkrankungen) kaum Auffälligkeiten zeigen. Unter Adjustierung der Faktoren Alter, Geschlecht, Bildungsniveau, Raucherstatus, Familienstand, Krankenversicherungsart und Body-Mass-Index (BMI) ergeben multivariate Analysen, dass sowohl die Anzahl der Grunderkrankungen als auch die Anzahl der gesundheitsbeeinflussenden Ereignisse signifikant negativ mit dem EQ-5D-Wert sowie dem körperlichen SF-8-Summenscore assoziiert sind.
Auch die Faktoren weibliches Geschlecht, Alter und BMI wirken sich negativ auf die Lebensqualität aus. Bei der mentalen Skala des SF-8 spielt allein die Anzahl der Ereignisse, nicht aber die der chronischen Erkrankungen, eine signifikant (negative) Rolle für die Höhe der Lebensqualität. Bei Einschluss der einzelnen Grunderkrankungen bzw. Ereignisse in die Regression zeigt sich der stärkste Einfluss durch kardiovaskuläre Ereignisse in den vergangenen 2 Jahren auf den EQ-5DGesamtwert. Hinzu kommen eine PAVKGrunderkrankung, zerebrovaskuläre Ereignisse und Krankenhausaufenthalte aufgrund eines Gelenkersatzes, chronisch obstruktiver Lungenerkrankung (COPD) oder gastrointestinaler Blutungen. Hypertonie, Diabetes oder Störungen des Fettstoffwechselsystems haben hingegen keinen direkten signifikanten Einfluss [32].

Insgesamt ist festzuhalten, dass Multimorbidität einen signifikant negativen Effekt auf die Lebensqualität älterer Personen hat - insbesondere auf die körperliche Komponente. Die Ergebnisse können nun einen Beitrag leisten, ein umfassendes Verständnis der Auswirkungen von Multimorbidität auf die gesundheitsbezogene Lebensqualität der größer werdenden Gruppe älterer Menschen zu erlangen, um so umfassende und übergreifende Konzepte zur bedarfsgerechten Behandlung zu etablieren.

\section{Auswirkungen von Multimorbidität auf die gesund- heitsassoziierten Kosten}

Für eine optimale Allokation von knappen Ressourcen ist ein umfassendes Bild der Auswirkungen von Multimorbidität auf die gesundheitsassoziierten Kosten von hoher Relevanz. Während im Rahmen eines systematischen Reviews lediglich eine Studie für den deutschen Versorgungskontext identifiziert werden konnte, liefert die Literatur vor allem Studien aus dem US-amerikanischen Raum, die eine Verbindung zwischen Multimorbidität und Kosten aufzeigen. Es ist übergreifend festzustellen, dass eine zunehmende Anzahl von simultanen Erkrankungen mit steigender Kostenintensität assoziiert ist. Nicht abschließend geklärt ist dabei allerdings die Frage, ob sich die Kostenzuwächse mit zunehmender Multimorbidität linear oder exponentiell entwickeln. Acht der insgesamt eingeschlossenen 14 Studien zeigen einen näherungsweise linearen Kostenzuwachs bis zu einem (Multi-)Morbiditätswert von 2 bis 3 an. Drei weitere Studien deuten dagegen für diesen Bereich auf eine exponentielle Zunahme der Krankheitskosten mit zunehmender Morbidität hin. Aus den restlichen drei Studien lassen sich keine eindeutigen Aussagen ableiten. Bemerkenswert ist, dass keine Untersuchung auf ein abnehmendes Wachstum der Kosten in diesen Morbiditätsbereichen hinweist. Für darüber hinausgehende Morbiditätslevel ( $\mathrm{ab}$ einem Wert von 3 Erkrankungen bzw. 3 Punkten auf einer Indexskala) lassen sich dagegen kaum generalisierbare Aussagen aus den verfügbaren Studien ableiten [33].

Um den Einfluss des Ausmaßes von Multimorbidität auf den gesundheitsbezogenen Ressourcenverbrauch und die damit assoziierten Kosten von Personen im hohen Alter abzuschätzen, wurden die Daten des 7-Jahres-Follow-ups der getABI-Kohorte genutzt. Dabei wurden 1937 Personen im Alter von über 72 Jahren eingeschlossen. Die Teilnehmer wurden zu der persönlichen Morbiditätssituation und der Inanspruchnahmehäufigkeit von Gesundheitsleistungen befragt. Zur monetären Bewertung des erfragten Ressourcenverbrauchs wurden „unit costs“ für alle berücksichtigten Leistungen ermittelt. Basierend auf einem kumulativen Multimorbiditätsindex erfolgte eine Abschätzung des Einflusses von Multimorbidität auf verursachte Kosten. Die durchschnittlichen Gesamtkosten nehmen bei der getABI-Kohorte mit steigender Multimorbidität von 1250 EUR bei keiner Erkrankung bis 6862 EUR bei 10 und mehr Erkrankungen linear $\mathrm{zu}$, wobei sich jedoch die Kostenanteile der verschiedenen Leistungsbereiche fundamental verschieben. Während bei niedrigen Multimorbiditätsstufen (bis 4 parallele Erkrankungen) vor allem ambulante Kosten und Arzneimittelkosten die Höhe der Gesamtkosten bestimmen, sind es vorwiegend stationäre Kosten bei höheren Multimorbiditätsgraden (• Abb. 1). So erhöhen sich die ambulanten Kosten zwischen Morbiditätsstufe 1 und 10 um den Faktor 2 (Ärz- 


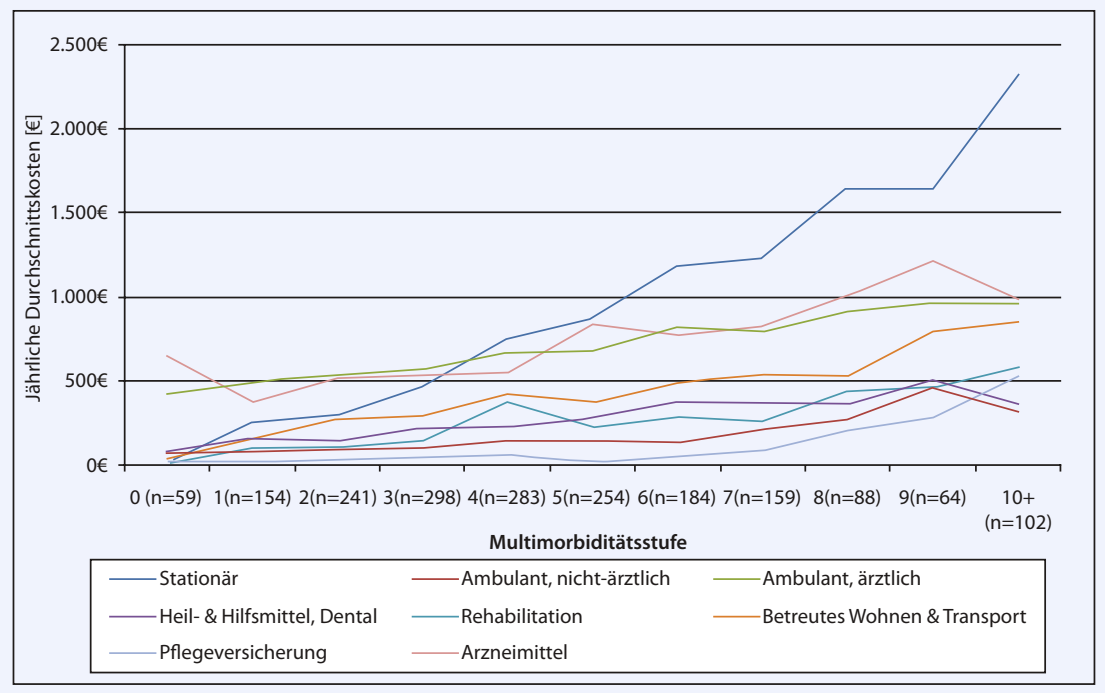

Abb. $1 \Delta$ Kostenanteile der Leistungsbereiche in EUR über Multimorbiditätsstufen

te) bzw. 4,4 (nichtärztliche Leistungsanbieter), die stationären Kosten jedoch um das 9,8-Fache. Eine regressionsanalytische Untersuchung zeigt, dass eine zusätzlich parallel auftretende Erkrankung mit einem Kostenanstieg von 563 EUR assoziiert ist. In dem Modell wirkt einzig die Multimorbidität bemerkenswert auf die Kosten, während Geschlecht, Alter, Bildung, BMI und Versichertenstatus keine signifikanten Einflussparameter darstellen. Allerdings muss angemerkt werden, dass das Regressionsmodell lediglich 11,5\% der Differenzen der Kostenhöhe erklären kann [46].

Die Analysen liefern dabei zur Literatur weitgehend deckungsgleiche Ergebnisse. So bewegen sich die Durchschnittskosten der Studienpopulation mit 3315 EUR in einem ähnlichen Bereich wie in der Studie von Heinrich [24], die ebenfalls für den deutschen Versorgungskontext 3730 EUR ermittelt. Auch die Ergebnisse zu den Ressourcenverbräuchen, z. B. für Hausarztbesuche ( $97,7 \%$ aller Studienteilnehmer hatten mindestens einen Hausarztbesuch) oder die durchschnittliche Anzahl an eingenommenen Medikamenten $(5,4)$, finden in der Berliner Altersstudie Entsprechungen in ähnlicher Größenordnung (93\% Hausarztbesuche, 6 Medikamente; [42]).

Ein detailliertes Verständnis der Kosteneffekte von bestimmten Multimorbiditätsmustern kann zu einer verbesserten Transparenz über die wichtigsten Kostentreiber im deutschen Gesundheitswesen führen. Daraus lässt sich ableiten, welche Erkrankungen bzw. Kombinationen vermehrt finanzielle Unterstützung zur Prävention, Erforschung und Behandlung benötigen. Im Hinblick auf die Allokation von Gesundheitsdienstleitungen und Ressourcen sollte es vor diesem Hintergrund das Ziel sein, Maßnahmen für die Krankheiten durchzuführen, die ein hohes Potenzial an Effizienz- und Einspareffekten bei den direkten und indirekten Kosten erwarten lassen. Hierfür bedarf es weiterer Forschung zu den Auswirkungen chronischer (Mehrfach-)Erkrankungen und deren Kombination, um dieses Wissen gezielt im Rahmen von Effizienzverbesserungen und in klinische Managementstrategien integrieren zu können.

\section{Schlussbemerkung}

In einer Gesellschaft des längeren Lebens und der damit einhergehenden chronifizierten Krankheitslast spielt Lebensqualität nicht nur für die Beurteilung medizinischer Interventionen, sondern (in Kombination mit Kosten) auch für Verteilungsfragen eine wesentliche Rolle. Die Ergebnisse aus Literatur und eigenen Analysen zeigen, dass Effekte auf Lebensqualität und Kosten nicht allein auf das Alter oder Geschlecht einer Person zurückgeführt werden können, sondern vielmehr auf die jeweilige Morbiditätssituation. Die Ergebnisse können zu einer erhöhten Transparenz im Bezug auf die Auswirkungen von Multimorbidität auf die Lebensqualität sowie die gesundheitsassoziierten Kosten im Alter führen.

\section{Ausblick auf die folgende Förderphase}

Auf Basis der Ergebnisse und Erfahrungen der ersten Förderphase setzt der Verbund PRISCUS seinen Schwerpunkt in der Folgeförderung auf Interventionsstudien. Im sportmedizinischen Folgeprojekt wird das bereits entwickelte und auf Machbarkeit getestete Übungs- und Bewegungsprogramm HOMEfit auf Wirksamkeit hin geprüft. Zu diesem Zweck werden 210 Patienten aus der hausärztlichen Versorgung zum neuen Bewegungsprogramm oder zu herkömmlichen Empfehlungen zu körperlicher Bewegung randomisiert und über insgesamt 12 Wochen unterstützt und nachbeobachtet. Das primäres Ziel der Studie ist zu prüfen, ob in Bezug auf sportmedizinische Assessmentparameter durch das Programm HOMEfit eine funktionelle Verbesserung zu erzielen ist.

In einer großen, clusterrandomisierten Studie wird außerdem ein Interventionspaket zur Reduktion potenziell inadäquater Medikation bei Älteren (RIME-Studie) evaluiert. Die Intervention wird neue pharmakologische Empfehlungen zur Vermeidung potenziell inadäquater Medikamente auf Basis der PRISCUS-Liste für den Hausarzt, aber auch für das gesamte Praxisteam enthalten sowie Hinweise zu relevanten Arzneimittelinteraktionen und Dosierungsfehlern geben. Die funktionelle Sicht auf den Patienten bleibt durch Beibehaltung eines geriatrischen Assessments erhalten. Das in der ersten Förderphase eingesetzte STEP-Assessment wird zur besseren Durchführbarkeit und Akzeptanz bei den Anwendern vereinfacht und modifiziert werden. Schließlich werden die Folgen der komplexen Intervention sowohl auf die Lebensqualität als auch auf mögliche gesundheitsökonomische Effekte hin untersucht.

Mit den Ergebnissen aus den beiden genannten Studien, aber auch mit Ergebnissen und Erfahrungen vieler weiterer Bereiche trägt der Forschungsverbund PRISCUS dazu bei, neue Ansätze zu Prävention, Diagnostik und Therapie bei Multimorbidität aufzuzeigen und Evidenz für therapeutische Interventionen im Kon- 
text von Multimorbidität zu erarbeiten. Damit wird die Perspektive geschaffen, in der Zukunft durch Anpassung der Versorgungsstruktur nach Vorbild des Chronic Care Modells ein verbessertes Management von Patienten mit Multimorbidität $\mathrm{zu}$ erreichen.

\section{Korrespondenzadresse \\ Dr.U. Thiem*}

Klinik für Altersmedizin und Frührehabilitation Marienhospital Herne, Klinik Börnig

Ruhr-Universität Bochum

Widumer Str. 8, 44627 Herne

ulrich.thiem@ruhr-uni-bochum.de

Danksagung. Die Mitarbeiter des Forschungsverbunds PRISCUS bedanken sich herzlich beim Förderer, dem Bundesministerium für Bildung und Forschung, sowie dem Projektträger DLR. Danken möchten wir auch den Mitgliedern des wissenschaftlichen Beirats für wertvolle Hilfen und Anregungen sowie unserer Kooperationspartnern für die gute Zusammenarbeit. Schließlich gilt unser Dank auch den Teilnehmern der verschiedenen Studien des PRISCUS-Verbunds, ohne deren Bereitschaft zur Teilnahme Forschung dieser Art nicht möglich wäre.

Interessenkonflikt. Der korrespondierende Autor gibt an, dass kein Interessenkonflikt besteht. S. HoltNoreiks und P.A. Thürmann erhielten ein Vortragshonorar von der Firma Rottapharm Madaus GmbH.

\section{Literatur}

1. Beers MH (1997) Explicit criteria for determining potentially inappropriate medication use by the elderly. Arch Intern Med 157:1531-1536

2. Berg AO, US Preventive Services Task Force (2002) Behavioral counseling in primary care to promote physical activity: recommendations and rationale. Am Fam Physician 66:1931-1936

3. Bodenheimer T, Wagner EH, Grumbach K (2002) Improving primary care for patients with chronic illness: the chronic care model, part 2. JAMA 288(15):1909-1914

4. Bongue B, Naudin F, Laroche ML et al (2009) Trends of the potentially inappropriate medication consumption over $10 \mathrm{yrs}$ in older adults in the east of France. Pharmacoepidemiol Drug Saf 18:11251133

5. Boyd CM, Boult C, Shadmi E et al (2007) Guided care for multimorbid older adults. Gerontologist 47(5):697-704

6. Boyd CM, Darer J, Boult C et al (2005) Clinical practice guidelines and quality of care for older patients with multiple comorbid diseases. JAMA 294:716-724

7. Buck MD, Atreja A, Brunker CP et al (2009) Potentially inappropriate medication prescribing in outpatient practices: prevalence and patient characteristics based on electronic health records. Am J Geriatr Pharmacother 7:84-92

8. Charlson ME, Charlson RE, Peterson JC et al (2008) The Charlson comorbidity index is adapted to predict costs of chronic diseases in primary care patients. J Clin Epidemiol 61(12):1234-1240
9. Chodzko-Zajko WJ, Proctor DN, Singh MAF et al (2009) Exercise and physical activity for older adults. Med Sci Sports Exerc 41:1510-1530

10. Cohen HJ, Feussner JR, Weinberger M et al (2002) A controlled trial of inpatient and outpatient geriatric evaluation and management. N Engl J Med 346:905-912

11. Department of Health: Single Assessment Process. London. http://collections.europarchive.org/ tna/20100509080731/http://dh.gov.uk/en/SocialCare/Chargingandassessment/SingleAssessmentProcess/index.htm. Zugegriffen: 1. Aug. 2011

12. Deveugele $M$, Derese A, Brink-Muinen A van den et al (2002) Consultation length in general practice: cross sectional study in six European countries. BMJ 325:472

13. Epping-Jordan JE, Pruitt SD, Bengoa R, Wagner EH (2004) Improving the quality of health care for chronic conditions. Qual Saf Health Care 13:299-305

14. Fialová D, Topinková E, Gambassi G et al (2005) Potentially inappropriate medication use among elderly home care patients in Europe. JAMA 293:1348-1358

15. Fick DM, Cooper JW, Wade WE et al (2003) Updating the Beers criteria for potentially inappropriate medication use in older adults. Results of a US consensus panel of experts. Arch Intern Med 163:2716-2724

16. Fiss T, Dreier A, Meinke C et al (2011) Frequency of inappropriate drugs in primary care: analysis of a sample of immobile patients who received periodic home visits. Age Ageing 40:66-73

17. Fletcher AE, Price GM, Ng ES et al (2004) Population-based multidimensional assessment of older people in UK general practice: a cluster-randomised factorial trial. Lancet 364:1667-1677

18. Fortin M, Bravo G, Hudon C et al (2006) Psychological distress and multimorbidity in primary care. Ann Fam Med 4:417-422

19. Frey I, Berg A, Grathwohl D, Keul J (1999) Freiburger Fragebogen zur körperlichen Aktivität - Entwicklung, Prüfung und Anwendung. Soz Praventivmed 44:55-64

20. Gallagher PF, Barry PJ, Ryan C et al (2008) Inappropriate prescribing in an acutely ill population of elderly patients as determined Beers' criteria. Age Ageing 37:96-101

21. Gensichen J, Muth C, Butzlaff M et al (2006) Die Zukunft ist chronisch: das Chronic-care-Modell in der deutschen Primärversorgung - Übergreifende Behandlungsprinzipien einer proaktiven Versorgung für chronisch Kranke. Z Arztl Fortbild Qualitatssich 100(5):365-374

22. getABI Study Group (2002) getABI: German epidemiological trial on ankle brachial index for elderly patients in family practice to detect peripheral arterial disease, significant marker for high mortality. VASA 31:241-248

23. Grobe T, Döring H, Schwartz FW (2009) GEK-Report ambulant ärztliche Versorgung. Asgard, Sankt Augustin

24. Heinrich S, Luppa M, Matschinger $\mathrm{H}$ et al (2008) Service utilization and health-care costs in the advanced elderly. Value Health 11(4):611-620

25. Hinrichs T, Brach M (2011) The general practitioner's role in promoting physical activity to older adults: a review based on program theory. Curr Aging Sci (E-pub ahead of print)

26. Hinrichs T, Bucchi C, Brach M et al (2011) Heimübungsprogramm für das hohe Lebensalter mit Unterstützung durch die hausärztliche Praxis ( $\mathrm{HO}$ MEfit) - von der Machbarkeit zur Evaluation. Dtsch Z Sportmed 62:195
27. Hinrichs T, Bucchi C, Brach M et al (2009) Feasibility of a multidimensional home-based exercise programme for the elderly with structured support given by the general practitioner's surgery: Study protocol of a single arm trial preparing an RCT [ISRCTN58562962]. BMC Geriatr 9:37

28. Hinrichs T, Moschny A, Klaaßen-Mielke R et al (2011) General practitioner advice on physical activity: analyses in a cohort of older primary health care patients (getABI). BMC Fam Pract 12:26

29. Hinrichs T, Trampisch U (2010) Körperliche Aktivität bei multimorbiden Patienten. Public Health Forum 18:29-30

30. Hinrichs T, Trampisch U, Burghaus I et al (2010) Correlates of sport participation among community-dwelling elderly people in Germany: a crosssectional study. Eur Rev Aging Phys Act 7:105-115

31. Hodek J, Ruhe A, Greiner W (2010) Gesundheitsbezogene Lebensqualität bei Multimorbidität. Gesundheitswesen 72(8):455-465

32. Hodek J, Ruhe A, Greiner W (2009) Gesundheitsbezogene Lebensqualität bei Multimorbidität im Alter. Bundesgesundheitsbl Gesundheitsforsch Gesundheitsschutz 52(12):1188-1201

33. Hodek J, Ruhe A, Greiner W (2010) Assoziation zwischen Multimorbidität und Krankheitskosten: eine systematische Übersichtsarbeit. PharmacoEconomics - German Research Articles 8(1):31-46

34. Holt S, Schmiedl S, Thürmann PA (2010) Potentially inappropriate medication in the elderly - PRISCUS list. Dtsch Arztebl Int 107:543-551

35. Holt S, Thiem U, Diederichs C et al (2010) Potentially inappropriate medication in two German elderly cohorts. DOI: $10.3205 / 10$ gaa22

36. Hudon C, Fortin M, Soubhi H (2008) Single risk factor interventions to promote physical activity among patients with chronic diseases - systematic review. Can Fam Physician 54:1130-7

37. Junius U, Fischer G (2002) Geriatrisches Assessment für die hausärztliche Praxis: Ergebnisse einer konzertierten Aktion aus 7 europäischen Ländern. Z Gerontol Geriatr 35:210-223

38. Junius U, Schulz C, Fischer G et al (2003) Das europäische Assessment im Praxistest: Ergebnisse aus der deutschen Machbarkeitsstudie. ZFA 79:620623

39. Kaplan MS, Newsom JT, McFarland BH, Lu LN (2001) Demographic and psychosocial correlates of physical activity in late life. Am J Prev Med 21:306-312

40. Laroche ML, Charmes JP, Merle L (2007) Potentially inappropriate medications in the elderly: a French consensus panel list. Eur J Clin Pharmacol 63:725731

41. Lechevallier-Michel N, Gautier-Bertrand M, Alpérovitch $A$ et al (2005) Frequency and risk factors of potentially inappropriate medication use in a community-dwelling elderly population: results from the $3^{\circ} \mathrm{C}$ Study. Eur J Clin Pharmacol 60:813-819

42. Linden M, Gilberg R, Horgas A et al (2010) Die Inanspruchnahme medizinischer und pflegerischer Hilfe im hohen Alter. In: Lindenberger U, Smith J, Mayer K, Baltes P (Hrsg) Die Berliner Altersstudie. 3. Aufl. Akademie Verlag, Berlin, S 499-520

43. McLeod PJ, Huang A, Tamblyn RM, Gayton DC (1997) Defining inappropriate practices in prescribing for elderly people: a national consensus panel. CMAJ 156:385-391

44. Moschny A, Platen P, Klaaßen-Mielke R et al (2011) Physical activity patterns in German elderly men and women: a cross-sectional study. BMC Public Health 11:559 
45. Moschny A, Platen P, Klaaßen-Mielke R, Hinrichs $T$ (2011) Barrieren für körperliche Aktivität im höheren Lebensalter - Analysen einer Kohorte älterer Hausarztpatientinnen und -patienten (getABI). Dtsch Z Sportmed 62:194

46. Nagl A, Witte J, Hodek J, Greiner W. Relationship between multimorbidity and direct healthcare costs in an advanced elderly population: results of the PRISCUS trial. Z Gerontol Geriatr (eingereicht)

47. Nelson ME, Rejeski WJ, Blair SN et al (2007) Physical activity and public health in older adults: recommendation from the American College of Sports Medicine and the American Heart Association. Circulation 116:1094-1105

48. Sandholzer $\mathrm{H}$, Hellenbrand W, Renteln-Kruse W et al (2004) STEP-standardized assessment of elderly people in primary care. Dtsch Med Wochenschr 129 (Suppl 4):183-226

49. Statistisches Bundesamt (2008) Gesundheit Krankheitskosten 2002, 2004 und 2006. Statistisches Bundesamt, Wiesbaden

50. Statistisches Bundesamt (2009) 12. koordinierte Bevölkerungsvorausberechnung. Statistisches Bundesamt, Wiesbaden

51. Statistisches Bundesamt/Deutsches Zentrum für Altersfragen/Robert-Koch-Institut (2009) Beiträge zur Gesundheitsberichterstattung, Gesundheit und Krankheit im Alter. Robert-Koch-Institut, Berlin

52. Steinhagen-Thiessen E, Borchelt M (1996) Morbidität, Medikation und Funktionalität im Alter. In: Mayer KU, Baltes PB (Hrsg) Die Berliner Altersstudie. Akademie Verlag, Berlin, S 151-183

53. Stuck AE, Siu AL, Wieland GD et al (1993): Comprehensive geriatric assessment: a meta-analysis of controlled trials. Lancet 342:1032-1036

54. Theile G, Winter A, Hummers-Pradier E, JuniusWalker U (2008) Was verbirgt sich hinter dem hausärztlichen geriatrischen Assessment? [What is hidden behind the geriatric assessment in general practice?] ZFA 84:16

55. Thiem U, Theile G, Junius-Walker U et al (2011) Prerequisites for a new health care model for elderly people with multimorbidity: the PRISCUS research consortium. Z Gerontol Geriatr 44(2):115-120

56. Tinetti ME, Bogardus ST, Agostini JV (2004) Potential pitfalls of disease-specific guidelines for patients with multiple conditions. N Engl J Med 351:2870-2874

57. Trampisch U, Platen P, Burghaus I et al (2010) Reliabilität des PRISCUS-PAQ - Fragebogen zur Erfassung körperlicher Aktivität von Personen im Alter von 70 Jahren und älter. Z Gerontol Geriatr 43:399-406

58. Trampisch U, Platen P, Burghaus I et al (2011) Validität des PRISCUS-PAQ - Fragebogen zur Erfassung körperlicher Aktivität von Personen im Alter von 70 Jahren und älter. Z Gerontol Geriatr (zur Publikation angenommen)

59. Trampisch U, Platen P, Moschny A, Hinrichs T (2011) Über die Eignung von Fragebögen zur Erfassung der körperlichen Aktivität älterer Erwachsener für den Einsatz in einer epidemiologischen Studie - Gewinnung von Hinweisen zur Neuentwicklung des PRISCUS-PAQ. Dtsch Z Sportmed:329-333

60. Uijen AA, Lisdonk EH van de (2008) Multimorbidity in primary care: prevalence and trend over the last 20 years. Eur J Gen Pract 14 (Suppl I):28-32

61. Vass M, Avlund K, Hendriksen C et al (2007) Preventive home visits to older people in Denmark why, how, by whom, and when? Z Gerontol Geriatr 40:209-216
62. Walter U, Schneider N, Bisson S (2006) Krankheitslast und Gesundheit im Alter. Bundesgesundheitsbl Gesundheitsforsch Gesundheitsschutz 49:537-546

63. Wurm S, Tesch-Römer C (2006) Gesundheit, Hilfebedarf und Versorgung. In: Tesch-Römer C, Engstler $\mathrm{H}$, Wurm S (Hrsg) Altwerden in Deutschland. VS, Wiesbaden, S 329-383 